\title{
Design of synthetic jet actuator based on FSMA composite
}

\author{
Yuanchang Liang ${ }^{\mathrm{a}}$, Yasuo Kuga ${ }^{\mathrm{b}}$, Minoru Taya ${ }^{\mathrm{a}}$ \\ ${ }^{a}$ Center for Intelligent Materials and Systems, Department of Mechanical Engineering, University of \\ Washington, Seattle, WA, USA \\ ${ }^{b}$ Department of Electrical Engineering, University of Washington, Seattle, WA, USA
}

\begin{abstract}
An improved version of the membrane actuator has been designed and constructed based on our previous diaphragm actuator. It consists of ferromagnetic shape memory alloy composite (FSMA) diaphragm and an electromagnet system. The actuation mechanism of the membrane actuator is the hybrid mechanism that we proposed previously. The high momentum airflow will be produced by the oscillation of the circular FSMA composite diaphragm driven by electromagnets close to its resonance frequency. This membrane actuator is designed for the active flow control technology on airplane wings. The active flow control (AFC) technology has been studied and shown that it can help aircraft improve aerodynamic performance and jet noise reduction. AFC can be achieved by a synthetic jet actuator injecting high momentum air into the airflow at the appropriate locations on aircraft wings. Due to large force and martensitic transformation on the FSMA composite diaphragm, the membrane actuator can produce $190 \mathrm{~m} / \mathrm{s}$ synthetic jets at $220 \mathrm{~Hz}$. A series connection of several membrane actuators is proposed to construct a synthetic jet actuator package for distributing synthetic jet flow along the wing span.
\end{abstract}

Keywords: shape memory alloy, synthetic jet, ferromagnetic, superelasticity, actuator, composite, active flow control, vibration, hybrid mechanism

\section{Introduction}

The control of air separation from a wing in flight is essential for the modern aircraft to improve its aerodynamic performance. It has been done by geometric changes of wings depending on the flying condition. Recently, active flow control (AFC) was proposed as an alternative to control the air separation, especially for the fixed-wing aircraft. It has been shown that active flow control technology can help aircraft improve aerodynamic performance, such as jet noise reduction and aerodynamic stall improvement by adding external energy into the flow field [1-11]. AFC can be achieved by injecting synthetic jet with high momentum air into the flow at the appropriate locations (near the point of airflow separation) on aircraft wings. It will reattach the flow, increase lift and reduce the drag. Instead of steady blowing synthetic jet, the oscillated synthetic jet has been shown that it is more efficient and reliable to delay the flow separation. AFC technology has been heavily investigated and applied on the wings of rotorcrafts, for example V-22 and VR-7 $[8,10]$, because the rotorcrafts experience a great penalty in payload and lift capability in hovering. There is a significant improvement to increase the capability using AFC by synthetic jet actuators. Currently, most of the synthetic jet actuators for AFC have been constructed based on piezoelectric materials to produce synthetic jet flow [8-11]. Some piezoelectric material based synthetic jet actuator can produce up to $100 \mathrm{~m} / \mathrm{s}$ with frequency range of $300 \mathrm{~Hz}$. However, this may not strong enough for higher Mach number flight. Therefore, we are seeking another design approach of a synthetic jet actuator based on ferromagnetic shape memory alloy (FSMA) composite and the hybrid mechanism, which can produce much stronger synthetic jet flow with high frequency.

Ferromagnetic shape memory alloy (FSMA) composite [12-14] is made of ferromagnetic material and superelastic grade shape memory alloy. The function of the former is to produce a large magnetic force by the electromagnet due to high magnetic field gradient, while that of the latter is to sustain large stress and induce larger reversible strain. The high magnetic field gradient can be introduced by the fast responsive electromagnet system. This driving mechanism on the FSMA composite is called the hybrid mechanism [15] which is based on the stress-induced martensitic phase transformation produced by applied magnetic field gradient, thus enhancing the displacement, as the stiffness of shape memory alloy reduces due to the martensitic phase transformation. We have designed and fabricated the membrane actuator for the synthetic jet actuator based on the hybrid mechanism, the FSMA composite diaphragm and an 
electromagnet system. This paper will present the finite element analysis and experimental results of the actuator system including the dynamic performance. The goal of the membrane actuator is to produce large force and reasonable stroke, hence, can be applied to active flow control technology.

(a)

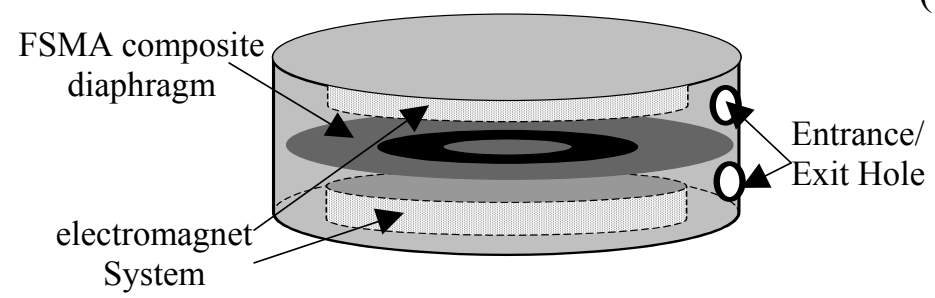

(b)

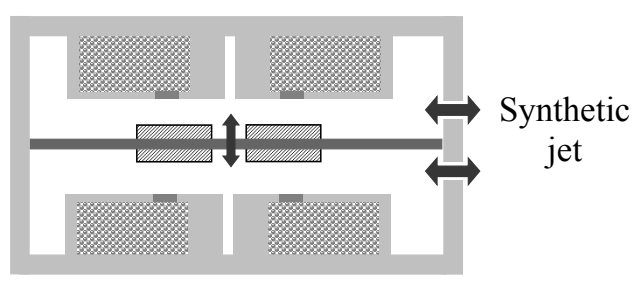

Figure 1 (a) the schematic of the membrane actuator, (b) the synthetic jet flow created by the oscillation of the FSMA composite diaphragm.

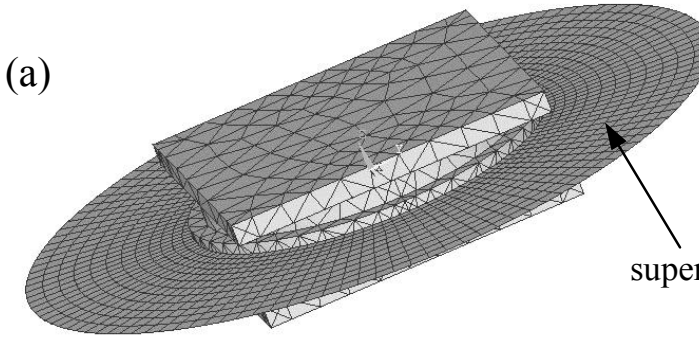

(b)

Figure 2 (a) the FEM model of the FSMA composite diaphragm, and (b) its size view

\section{Design and estimation of the membrane actuator system}

The membrane actuator consists of a FSMA composite diaphragm and an electromagnet system as shown in Fig. 1(a). The FSMA composite diaphragm is made of a superelastic grade NiTi thin plate and ferromagnetic soft iron pads. It will oscillate driven by the electromagnet system and create a jet flow (black arrow) through the entrance/exit hole, as shown in Fig.1(b). The electromagnet system includes soft iron yoke laminate, magnet wire coil and permanent magnet, which will provide large magnetic field gradient resulting in a large force on the composite diaphragm. The electromagnet system and the superelastic property of the NiTi plate will ensure the reversible and large deformation at a reasonable fast actuation speed. When the FSMA composite diaphragm vibrates close to its resonance frequency, its stroke can reach the maximum magnitude and produce strong synthetic jet flow, while the superelastic NiTi sheet can sustain large stresses without a permanent deformation. In order to achieve this operation, the resonance frequency of the composite diaphragm has to match closely with the magnetic field frequency response of the electromagnet system. Both the mechanical and electromagnetic finite element analyses are used for designing the optimum composite structure.

The dynamic analysis of the FSMA composite diaphragm is based on the ANSYS finite element analysis. Figure 2 (a) and (b) shows its ANSYS model and the side views of the composite diaphragm. The soft iron pads on each side of the composite diaphragm are square in order to obtain force as large as possible from the electromagnet system. Two spacers are used between the soft iron pad and the NiTi diaphragm as shown in Fig. 2(b). Spacer\#1 is a solid circular steel plate to maintain as a rigid body and to ensure the uniform stress distribution along the circumference on the NiTi diaphragm. This spacer also can be used to adjust the resonance frequency of the composite diaphragm depending on its dimension and weight, which will change the stiffness and mass of the structure. Spacer\#2 is made of aluminum and it is to prevent the contact between the soft iron pad and the NiTi sheet during the oscillation. The Young's modulus of $55 \mathrm{GPa}$, poisson ratio of 0.33 and density of $6500 \mathrm{Kg} / \mathrm{m}^{3}$ are used as the input data for the NiTi plate. The Young's modulus of 210GPa, poisson ratio of 0.33 and density of $7850 \mathrm{Kg} / \mathrm{m}^{3}$ are used for the soft iron pad. The Young's modulus of $70 \mathrm{GPa}$, poisson ratio of 0.33 and density of $2700 \mathrm{Kg} / \mathrm{m}^{3}$ are used for the aluminum pad. In the simulation, the boundary condition of the NiTi diaphragm is assumed to be totally constrained without rotation and slipping on its 
own diameter circumference and interface with the Spacer\#1 circumference. Because of the limitation of ANSYS FEA program which can only perform the frequency response calculation with a linear Young's modulus, the estimation is only valid before the stress-induced martensitic transformation (at $450 \mathrm{MPa}$ ). However, this analysis result gives us a designing guidance of the composite diaphragm frequency we can drive. Figure 3(a) shows the FEA results of the resonance frequency of the composite diaphragm as a function of the diaphragm thickness. The NiTi diaphragm is $64 \mathrm{~mm}$ in diameter and the size of the soft iron pad is $32 \mathrm{~mm} \times 30 \mathrm{~mm} \times 3 \mathrm{~mm}$. Spacer $\# 1$ has a size of $33 \mathrm{~mm}$ in diameter and $1 \mathrm{~mm}$ in thickness, and Spacer $\# 2$ has the size of $30 \mathrm{~mm}$ in diameter and $2 \mathrm{~mm}$ in thickness. It clearly shows that thicker the diaphragm, higher the resonance frequency becomes. The thickness of the soft iron pad also can change the resonance frequency of the entire composite diaphragm structure as well as the force induced by the electromagnet because larger the volume of pad, stronger the force. Figure 3(b) shows the results for the case of $0.3 \mathrm{~mm}$ thick diaphragm. The thicker the soft iron pad results in lowering the resonance frequency of the composite diaphragm although a larger force can be obtained. These data are very important for us to select a proper electromagnet system whose magnetic frequency response can match the mechanical frequency response of the composite diaphragm. The simulation results also suggest that the highest stress level occurs on the diameter circumference and the interface between the NiTi sheet and the pad. We have to assure that the stress level is near the onset of stress-induced martensitic transformation in order to obtain the large stroke of the diaphragm resulting in the strong synthetic jet flow velocity.

(a)

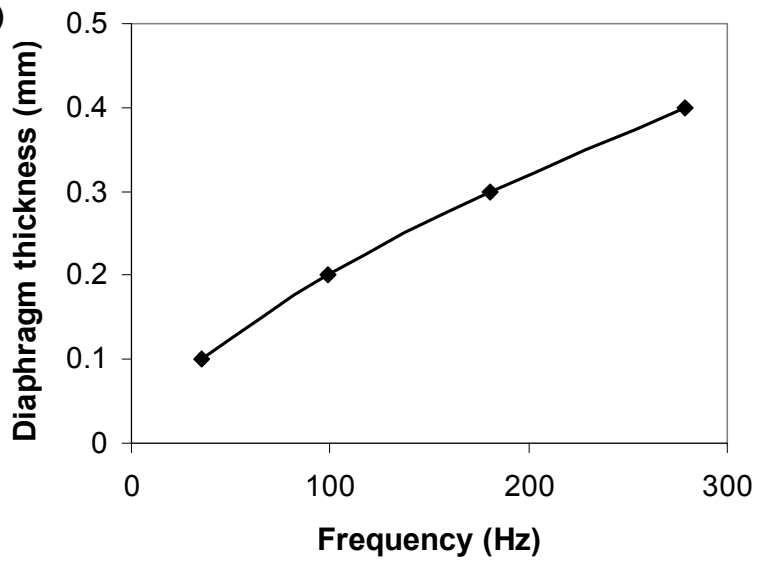

(b)

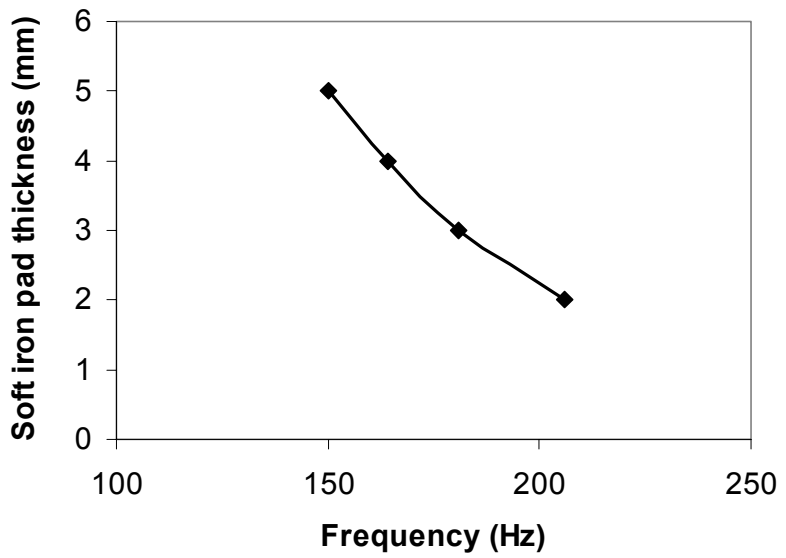

Figure 3 (a) the thickness of the NiTi diaphragm and (b) the soft iron pad thickness estimated by FEM as a function of the composite diaphragm resonance frequency.
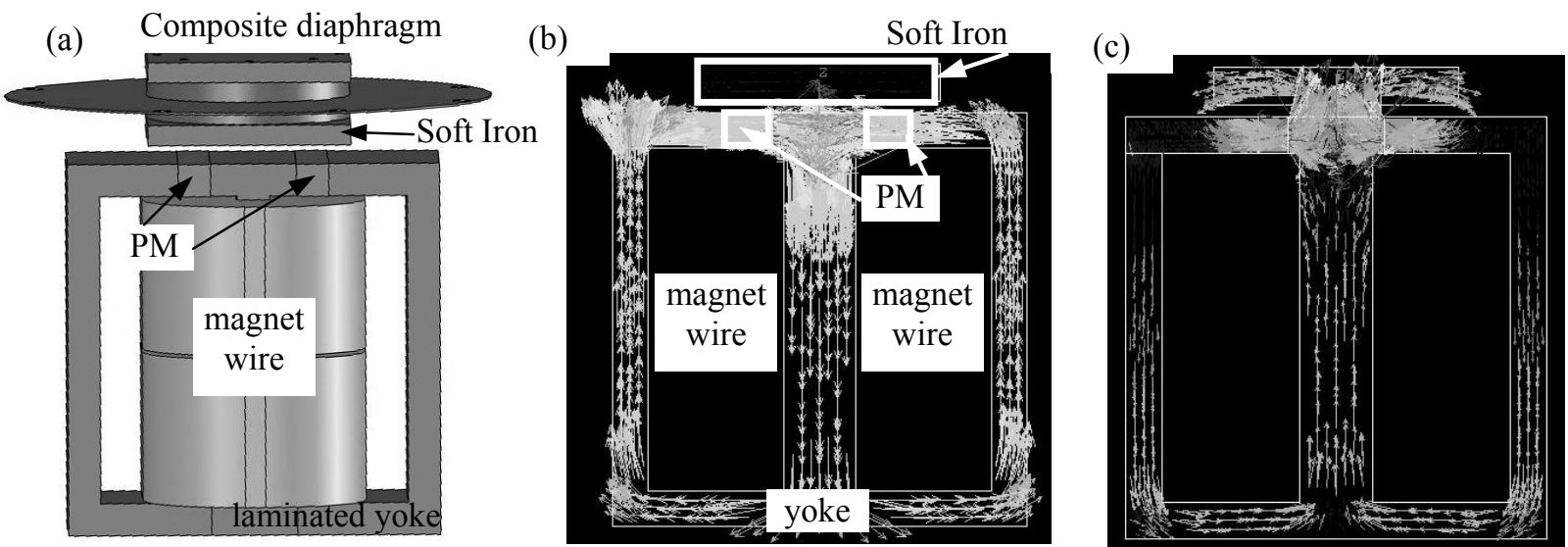

Figure 4 (a) model for 3D electromagnetic FEA, (b) FEA result of magnetic flux flows inside the yoke and no force is available on the soft iron when the power is off, and (c) magnetic flux goes through the soft iron pad and force is induced when the power is on 
The electromagnet system of the membrane actuator is based the concept of the hybrid magnet which consists of yoke, coil and permanent magnet [16]. It has been shown that the hybrid magnet system is more efficient and produces much larger force than the traditional solenoid magnet due to high magnetic field gradient [12-14]. Figure 4(a) shows the schematic of an electromagnet with the composite diaphragm for the three-dimensional electromagnetic FEA simulation. Both permanent magnets (PM) are polarized against each other and they are made of Neodium (with $\mathrm{Hc}$ of $840 \mathrm{KA} / \mathrm{m}$ ). As shown in Fig.4(a), the right permanent magnet produces a counter clockwise magnetic flux loop and the left permanent magnet produces a clockwise loop when the electromagnet is off. Both flux loops are close loops within the yoke and no magnetic flux goes into the soft iron pad which connects to the composite diaphragm. Therefore, no force is induced on the composite diaphragm. When the electromagnet is turned on, opposite directions of magnetic flux are formed against each original flux loops induced by each permanent magnet, Fig.4(c). The magnetic field flux will punch out of the yoke and go through the soft iron pad. This will result in a large pulling force on the composite diaphragm due to locally high magnetic field gradient. To ensure the high frequency response $(200 \sim 300 \mathrm{~Hz})$ and to increase the efficiency of the electromagnet system, the laminated yoke made of grain-oriented Fe-Si sheets $(0.15 \mathrm{~mm}$ thick $)$ is used. This is to eliminate the energy loss due to the eddy current effect and produce high magnetic field gradient resulting in strong force.

(a)

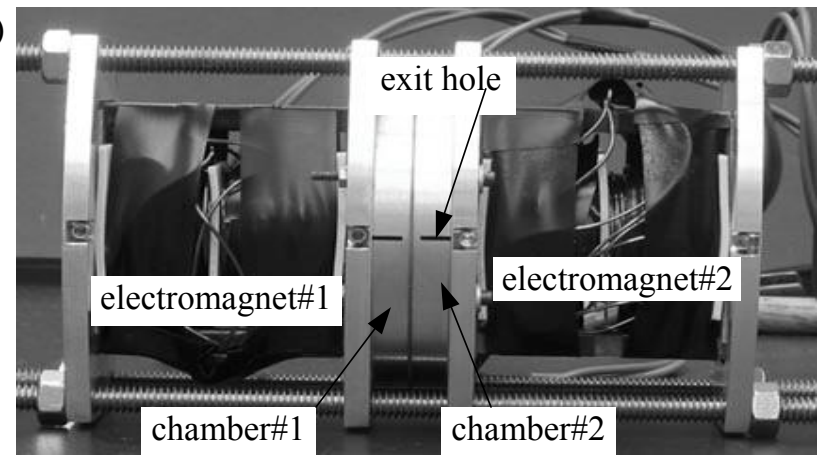

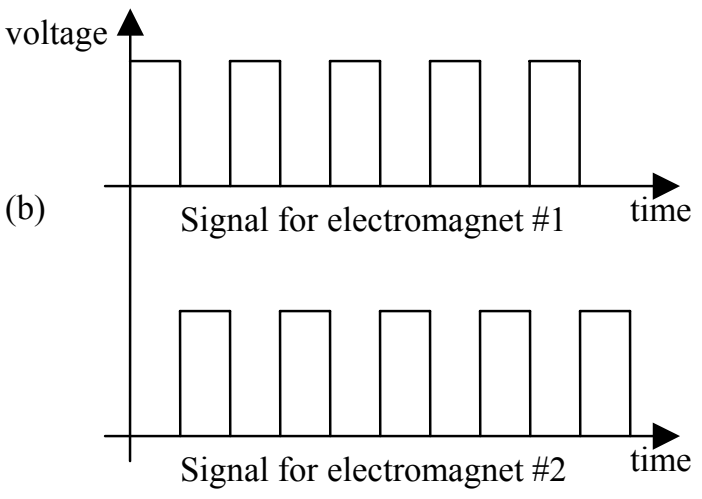

Figure 5 (a) the photo as assembled membrane actuator, and (b) the signals for both electromagnet units

\section{Dynamic testing results}

Figure 5(a) shows the photo the membrane actuator. The overall size of the actuator is $72 \mathrm{~mm} \times 85.6 \mathrm{~mm} \times 130 \mathrm{~mm}$ and the total weight is $1.36 \mathrm{Kg}$. It has 2 chambers in the center divided by the composite diaphragm. Each chamber has a $5 \times 1 \mathrm{~mm}$ hole as the exit for the synthetic jet flow. The superelastic NiTi sheet $(0.3 \mathrm{~mm}$ thick $)$ of the composite diaphragm is cut into a $72 \mathrm{~mm}$ in diameter circular diaphragm by the EDM machine. It will be clamped by 2 aluminum rings with $4 \mathrm{~mm}$ in width on both sides. Therefore the effective diameter of the composite diaphragm is $64 \mathrm{~mm}$. Soft iron pads and Spacer\#1 are made of 1018 low carbon steel with the size of $30 \mathrm{~mm} \times 32 \mathrm{~mm} \times 3 \mathrm{~mm}$ and $33 \mathrm{~mm}$ in diameter with $1 \mathrm{~mm}$ in thickness, respectively. Spacer\#2 has the dimension of $32 \mathrm{~mm}$ in diameter and $2 \mathrm{~mm}$ in thickness. The finite element analysis shows that the resonance frequencies of this composite diaphragms is $180 \mathrm{~Hz}$. Currently, the parts of the FSMA composite diaphragm and the aluminum rings are fixed by screws. Gaskets and silicon are applied to ensure the tight seal of the chamber. Figure 5(b) shows the input signals of the actuator system. Both signals for Electromagnet \#1 and Electromagnet \#2 are square wave controlled by a driver with a $180^{\circ}$ phase difference. The driver circuit of the membrane actuator is made of commercially available controller for stepping motor. No complex controller is needed.

The single electromagnet unit consists of laminated yoke, 2 electrical coils and 2 permanent magnets. The laminated yoke made by $0.15 \mathrm{~mm}$ thick Fe-Si sheets is to eliminate the eddy current effect for high frequency usage (up to $400 \mathrm{~Hz}$ ). In order to obtain a fast frequency response of circuit and a strong magnetic flux gradient, two electrical coils are electrically parallel connected but magnetically series connected. The magnetic polarization of both permanent magnets is opposite to each other and the magnetic flux flows inside the yoke as a close loop as shown in Fig.4(b). When the electrical current goes through both coils, the magnetic flux will punch out of the yoke located between permanent magnets and create a large magnetic flux gradient, Fig.4(c). Figure 6(a) shows the magnetic field responses of the single electromagnet unit as a function of frequency, where the solid lines are for 100turn coils and dot lines for 300 turn coils. 
The field was measured by a digital Teslameter (Group 3, DTM133). The Teslameter probe is sandwiched between the electromagnet surface and a soft iron pad. The electromagnet with 300 turn coils has poor frequency responses (dot lines) because the residual magnetic filed is very high when the electromagnet is off. This will cause the composite diaphragm not to return to its neutral position, resulting in a poor vibration response. The electromagnet with 100 turn coils (solid lines in Fig.6(a)) shows a very good dynamic performance. When there is no electrical current, the residual magnetic field is very small. When the power (4Amp, 25V) goes through coils, the magnetic field can reach $0.35 \mathrm{~T}$ with a slight decay as the frequency goes up to $300 \mathrm{~Hz}$. This will ensure that the membrane actuator can be operated up to $300 \mathrm{~Hz}$. The synthetic jet flow velocity from the membrane actuator is measured by the hotwire method (TSI IFA 100 with a platinum hot film probe). The probe is carefully located just at the exit hole and parallel to the length of the exit hole. Figure 6(b) shows the results of jet flow velocities as a function of frequency for the composite diaphragm with a $0.3 \mathrm{~mm}$ thick NiTi plate. The velocity increases as the frequency increases and shows the peak velocity of $190 \mathrm{~m} / \mathrm{s}$ at $220 \mathrm{~Hz}$. This trend is associated with the resonance frequency of the composite diaphragm structure and the frequency response of the electromagnet.
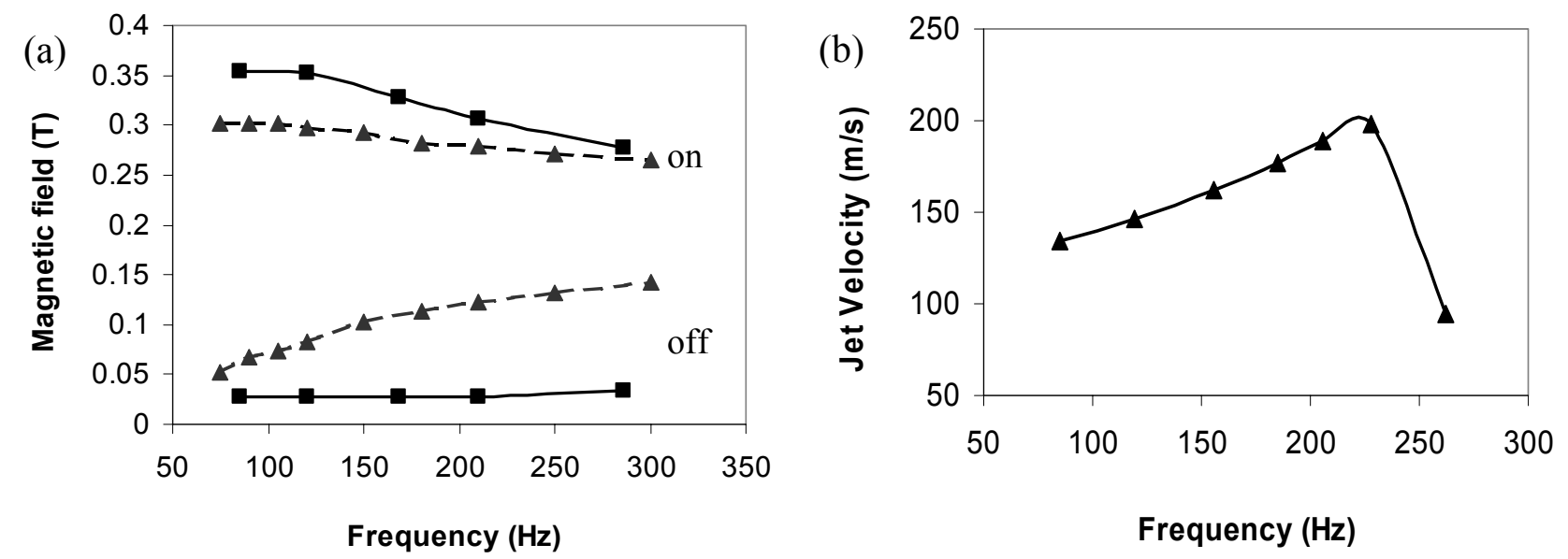

Figure 6 (a) the magnetic field responses as a function of frequency of a single electromagnet unit with the soft iron pad, and (b) the synthetic jet flow velocity as a function of frequency.

\section{Discussions}

The design concept of the membrane actuator is based on "hybrid mechanism", i.e. stress-induced martensitic phase transformation (SIM) caused by large force due to large magnetic field gradient, thus enhancing the displacement, as the stiffness of shape memory alloy reduces due to the martensitic transformation. Therefore, the information of the maximum stress level on the composite diaphragm is very important. The more displacement of the composite diaphragm due to the martensitic transformation, the larger the volume change we can obtain resulting in a stronger synthetic jet velocity. The displacement-frequency response was measured by a high-speed video camera system (KODAK Motion Corder Analyzer, Model 1000SR) as shown in Fig. 7(b). Due to the geometric limitation, the standard measurement of dynamic frequency response was not possible. The stroke reaches maximum of $1.86 \mathrm{~mm}$ at the frequency of $195 \mathrm{~Hz}$ which is smaller than the peak frequency for the maximum jet velocity as show in Fig.6(b). It may be due to the different level of air compression inside the chamber. Based on the theory of plate and shell [17], the maximum stress and the maximum displacement can be estimated by

$$
\begin{aligned}
& \sigma_{\text {max }}=k \frac{P}{h^{2}} \\
& w_{\text {max }}=k_{1} \frac{P a^{2}}{E h^{3}}
\end{aligned}
$$


Where $P$ is load, $h$ is thickness, $a$ is radius of diaphragm, $w$ is stroke and $E$ is Young's modulus. We can estimate the displacement of the composite plate before the onset SIM on the NiTi diaphragm. For the case of the composite diaphramg with a $0.3 \mathrm{~mm}$ thick NiTi sheet, when the stroke reaches $1.5 \mathrm{~mm}$, the NiTi diaphragm is at the onset of martensitic transformation (450MPa, Fig.7(b)). In addition, the FEA results show that the maximum stress reaches $510 \mathrm{MPa}$ when the stroke of the composite diaphragm is $1.86 \mathrm{~mm}$ at $190 \mathrm{~Hz}$, as shown in Fig. 7(a). Therefore, the FSMA composite diaphragm induces a strong synthetic jet flow when it goes into the stress-induced martensitc (SIM) transformation. However, the membrane actuator can produce a much stronger synthetic jet if more SIM induced on the composite diaphragm. Based on the testing results of the membrane actuator, we can calculate the performance of the FSMA composite diaphragm, such as energy density (ED), power density (PD) and the specific efficiency, which can be estimated by

$$
\begin{aligned}
& \mathrm{ED}=\frac{\text { work }}{V} \\
& \mathrm{PD}=\mathrm{ED} \times \text { Frequency }
\end{aligned}
$$

$$
\text { Specific efficiency }=\frac{\text { work } \times \text { frequency }}{\text { input power } \times M}
$$

where $\mathrm{V}$ is the FSMA composite volume and $\mathrm{M}$ is the total weight if actuator. Work can be estimated by the aera of the force-displacement curve of the composite diaphragm. Input power will be the input electrical power which can be calculated by area of the voltage-current curve of the actuator. For the current actuator results with a $0.3 \mathrm{~mm}$ thick composite diaphragm, ED is $30 \mathrm{KJ} / \mathrm{m}^{3}$, PD is $6000 \mathrm{KW} / \mathrm{m}^{3}$ and specific eifficiency is $12 \% / \mathrm{Kg}$. If more stroke of the composite diaphragm (faster the jet velocity) can be obtained due to SIM, both ED and PD will further increase. As shown in Eq.(4), higher the frequency response of the material, larger PD.
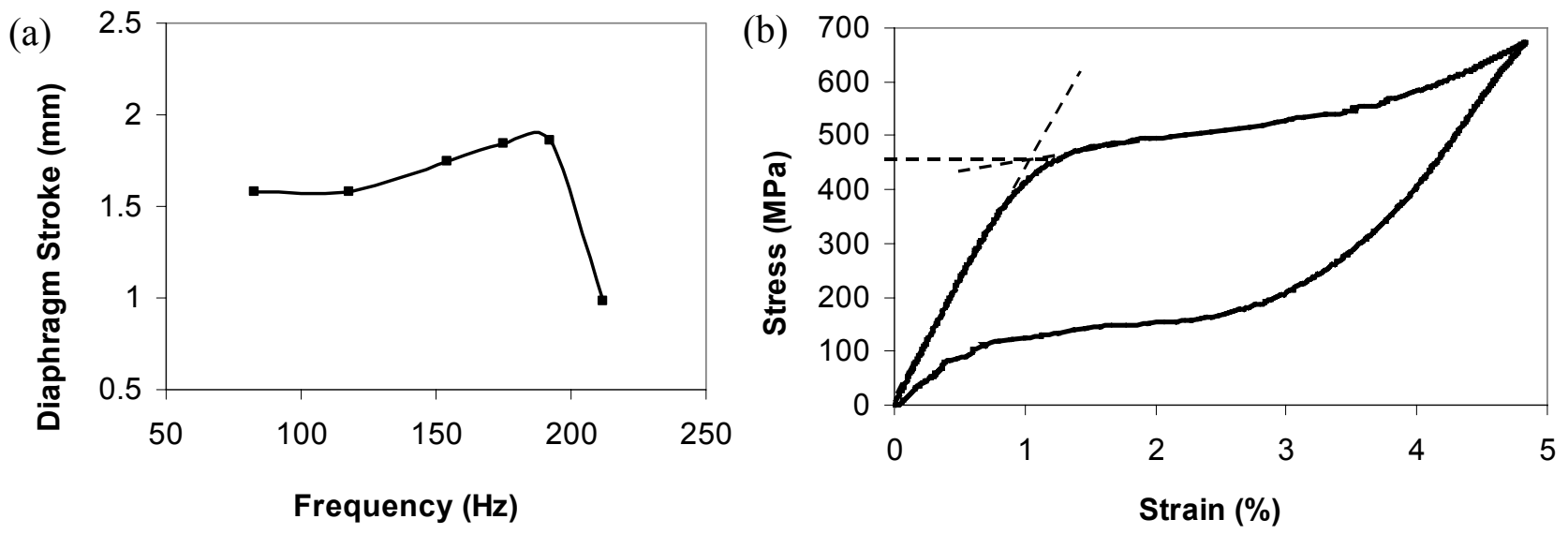

Figure 7 (a) the experimental result of the FSMA composite diaphragm stroke as a function of frequency, and (b) the stress-strain curve of the $0.3 \mathrm{~mm}$ thick NiTi superelastic sheet

\section{Synthetic jet actuator package}

Several units of membrane actuators can be series connected as a synthetic jet actuator package, as shown in Fig.8(a) which consists of 4 electromagnets and 3 FSMA composite diaphragms. The overall size of the package is about $72 \mathrm{~mm} \times 85.6 \mathrm{~mm} \times 300 \mathrm{~mm}$ with the weight of $3 \mathrm{Kg}$. This package can be integrated in the wing box to distribute the synthetic jet flow along the wing span, Fig.8(b). When Electromagnet \#1 and \#3 are turned on (Fig.9(a)), Diaphragm A and $\mathrm{C}$ will be attracted and move to left, and Diaphragm B will move to right. Therefore, Chamber A1, B3 and C3 will create the jet flow through out the chambers. Similarly, when Electromagnet \#2 and $\# 4$ are turned on (Fig.9(b)), Chamber A2, B2 and C4 will create synthetic jet flow. During the operation, two of the electromagnets (\#2 and \#3) in 
the center of the package will drive two composite diaphragms simultaneously. This can be achieved because the electromagnet based on the hybrid magnet concept can attract ferromagnetic material on both side of the electromagnet [14]. Therefore, less electromagnet units are needed to drive composite diaphragms in a package.

(a)

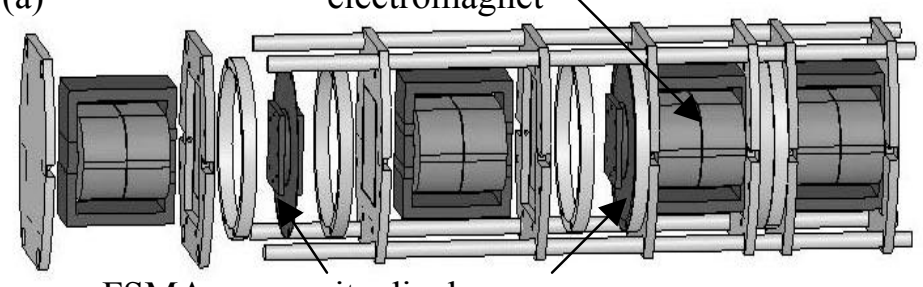

FSMA composite diaphragm (b)

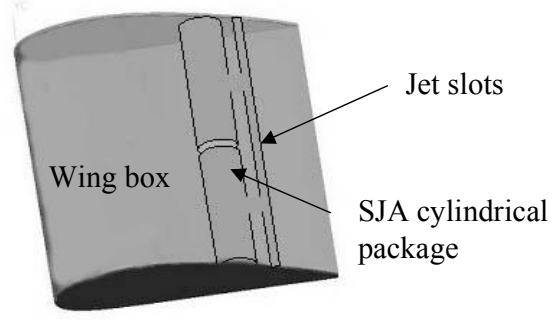

Figure 8 (a) the schematic of the synthetic jet actuator package, and (b) integration within the wing box

(a)

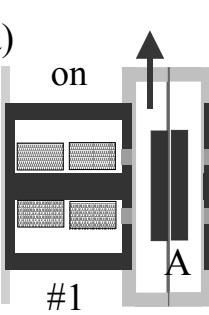

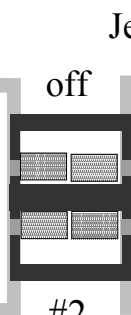

\#2

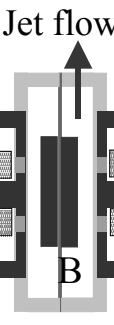

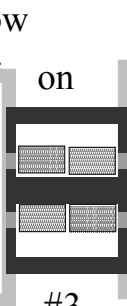

\#3

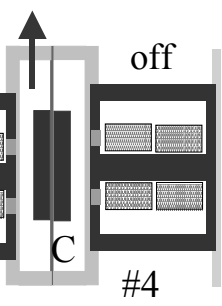

(b)

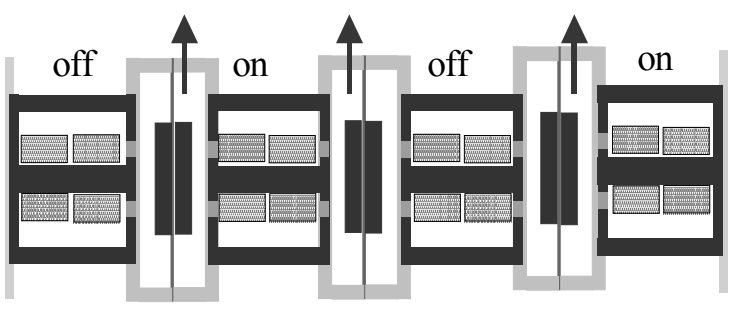

Figure 9 (a) and (b) the operating sequence of the synthetic jet actuator package

\section{Summary}

An improved design of membrane actuator based on the FSMA composites and hybrid mechanism for active flow control successfully shows a very good dynamic performance. It produces the maximum jet velocity of $190 \mathrm{~m} / \mathrm{s}$ at 220 Hz. The FSMA composite diaphragm oscillates at high frequency driven by the electromagnet system which provides high magnetic field gradient and strong force. The composite diaphragm goes into the stress-induced martensitic phase transformation produced by applied magnetic field gradient, thus enhancing the stroke and producing strong synthetic jet velocity. A series connection of several units of membrane actuators is also proposed for distributing the synthetic jet flow along the wing span.

\section{Acknowledgements}

This study was supported by DARPA-ONR contract (N-00014-00-1-0520). Dr. John Main of DARPA and Dr. Roshdy Barsoum of ONR are the program monitors. The present authors are thankful to Boeing Company (Mr. Ed White and Dr. Dale Pitt) for their advice on synthetic jet actuator design and also to Hitachi Metals (Mr. Masuzawa) for their assistance in providing ring-shaped permanent magnets, and also Mr. Suga of Nippon Cross Atueu and Mr. Kise of Kantoc in providing the superelastic NiTi plates. The acknowledgement also goes to Professor F. Gessner of Department of Mechanical Engineering in University of Washington who helped us in designing the hot wire measurement set up for jet velocity.

\section{References}

1. L. Pack and R. Joslin, "Overview of Active flow control at NASA Langley Research Center", Proceedings of SPIE, 3326, pp. 202-213, 1999.

2. A. Siefert, T. Bachar, D. Moss, M. Shepshelovich, I. Wygnanski, "Oscillatory Blowing: A Tool to Delay BoundaryLayer Separation”, AIAA Journal vol 31, No. 11, 1996, pg 2052-2060. 
3. A. Siefert, A. Darabi, I. Wygnanski, "Delay of Airfoil Stall by Periodic Excitation", AIAA Journal of Aircraft, Vol, No. 4, July 1996, pg 691-698.

4. A. Seifert, and L.G. Pack, Oscillatory control of separations at high Reynolds numbers, AIAA 98-0214, 1998.

5. I. Wygnanski, Boundary Layer and Flow Control by Period Addition of Momentum (Invited)", AIAA 97-2117, 1997.

6. M. Hites, H. Nagib, T. Bacher and I. Wygnanski, "Enhanced Performance of Airfoils at Moderate Mach Numbers Using Zero-Mass Flux Pulsed Blowing," AIAA Paper 2001-0734, 39th Aerospace Sciences Meeting and Exhibit, Reno, NV, Jan. 12-15, 2001

7. A. Honohan, M. Amitay and A. Glezer, "Aerodynamic Control Using Synthetic Jet", AIAA Fluids, June 2000, AIAA 2000-2401, 2000.

8. H. Nagib, J. Kiedaisch, D. Greenblatt, I. Wygnanski and A. Hassan, Effective flow control for rotorcraft applications at flight mach numbers, AIAA-2974, 2001

9. A. Hassan, H. Nagib, I. Wygnanski, "Oscillatory jets - Benefits and numerical modeling issues", AHS International, 58th Annual Forum Proceedings - Volume I, Montreal, Canada; 11-13 June 2002. pp. 711-731. 2002

10. F. T. Calkins, J.H. Mabe, J.P. Smith, and D.J. Arbogast, The Boeing Company Phantom Works, "Low frequency $(\mathrm{F}+=1.0)$ multilayer piezopolymer synthetic jets for active flow comtrol", AIAA paper, 2002-2823.

11. R. Bryant, R. Fox, J. Lachowicz, and F. Chen, "Piezoelectric synthetic jets for aircraft control surfaces", Proceedings of SPIE, 3674, pp. 220-227, 1999.

12. T. Wada and M. Taya, "Spring-based actuators", Proceedings of SPIE, 4699, pp. 294-302, 2002,

13. V.J. Cheng, M. Taya, J.K. Lee, M. Kusaka, T. Wada, "Design of torque actuator based on ferromagnetic shape memory alloy composite", Proc. SPIE, 5390, p. 309-316, 2004.

14. Y. Liang, M. Taya, Y. Kuga, "Design of diaphragm actuator based on ferromagnetic shape memory alloy composite", Proc. SPIE, Smart Structure and Material 2003: Industrial and Commercial Applications of Smart Structure Technologies, 5054, p.45-52, 2003.

15. Y. Liang, T. Wada, T. Tagawa and M. Taya, "Model Calculation of Stress-Strain Relationship of Polycrystalline FePd and 3D Phase transformation Diagram of Ferromagnetic Shape memory Alloys", Proceedings of SPIE, 4699, pp. 206-216, 2002.

16. K. Oguri, Y. Ochiai, Y. Nishi, S. Ohino and Y. Uchida, Extended Abstracts of $9^{\text {th }}$ Intelligent Materials Forum, Mitoh Science and Tech, Tokyo, March 16, pp.24-25, 2000.

17. S. Timoshenko, and S. Woinowsky-Krieger, Theory of plates and shells, McGraw-Hill, New York, $2^{\text {nd }}$ Edition, pp. $61-62$. 